\title{
PERCEPCIÓN Y DECISIÓN. CONSIDERACIONES TEÓRICAS ACERCA DE LA RELACIÓN ENTRE PERCEPCIÓN SOCIAL DE INSEGURIDAD Y CONDUCTA DELICTIVA*
}

\section{Perception and decision. Theoretical considerations on the relationship between social perception of insecurity and criminal behavior}

\author{
Juan Ignacio Piña Rochefort ${ }^{* *}$
}

\begin{abstract}
Resumen: El artículo aborda la discusión respecto a la toma de decisiones, las cuales estarían mediadas por las percepciones que de la realidad tiene cada sujeto. Estas decisiones, por tanto, se adoptan construyendo una realidad sobre la base de información deficiente. Esta premisa afecta las decisiones que en materia de política criminal y de seguridad pueden adoptarse, de modo que no necesariamente ellas son un correlato de lo que realmente ocurre, sino más bien una respuesta a lo que se percibe. La incidencia que tiene la percepción de algo en la decisión sería crucial, porque es el primer elemento que se toma en cuenta para la construcción del conocimiento y la generación de conceptos.
\end{abstract}

Palabras clave: percepto - juicio conceptual - razonamiento - construcción social percepción de inseguridad.

\begin{abstract}
The article explores the discussion regarding individual decision-making, which tends to be mediated by each subject's perception of reality. These decisions, therefore, are taken based on a deficient or incomplete information about reality. This affects decision in criminal and security policy too, because such policies are not necessarily a correlate of what actually happens, but rather respond to what is perceived. The relevance that a perception of something has in the decision about it would be crucial, because is the first element taken into account during knowledge- and concept-building.
\end{abstract}

Keywords: percept - conceptual judgement - reasoning - social construction perception of insecurity.

\section{Percepción y mundos}

Si bien es una de las expresiones más sugerentes y complejas en otras áreas de la ciencia, la noción del "percepto" resulta relativamente lejana a las ocupaciones del Derecho Penal y de la Política Criminal. Tampoco ha tenido un tratamiento o recepción contundente en los modelos de diseño de políticas públicas referidas a la

\footnotetext{
* Conferencia dictada en el 1 ${ }^{\text {er }}$ Encuentro Estudiantil de Derecho Penal y Política Criminal 2011. Universidad de Chile.

**A Abogado. Doctor en Derecho por la Universidad de Navarra, España. Profesor de Derecho Penal en la Universidad de los Andes. Correo electrónico: ipinaroc@uandes.cl
} 
Piña - Percepción y decisión. Consideraciones teóricas...

criminalidad. Sin embargo, la noción del perceptum, esto es, aquel objeto percibido pero no como es sino tal y como es percibido por el observador, ${ }^{1}$ puede ofrecernos más rendimiento del que usualmente hemos asumido. Peirce lo definía como "lo que estamos viendo antes de saber lo que estamos viendo", previo a la intervención de la "abducción" como inicial hipótesis de lo percibido. A partir de los datos ahí obtenidos se realiza un "juicio perceptual" que requiere de una búsqueda en la memoria asociativa, es decir, una revisión de aquello que ya se encuentra archivado a partir de la experiencia anterior y que además está mediado contextualmente por la cultura y por el momento histórico que opera como base de decodificación. Ello lo presenta como un mundo semiótico posible y constituye un instrumento imprescindible para la interpretación de lo percibido.

Con aproximaciones de esta naturaleza no solo se reconoce la relevancia de lo individual en el proceso sino que además se provee un enlace directo e inmediato con la realidad. ${ }^{2}$ Por eso cuando Peirce reconoce la contribución kantiana a la relación entre percepto y concepto, señala como uno de sus principales problemas el hecho de establecer una separación demasiado tajante entre la percepción y el razonamiento. Kant asumía que el segundo comenzaba una vez que el primero (la observación) había acabado. ${ }^{3} \mathrm{Y}$ aquí es donde comienza a ponerse sobre la mesa que así como la observación tiene un papel que cumplir en el razonamiento, la propia facultad receptiva involucra también un elemento intelectual (conceptos). ${ }^{4-5}$

No es posible entrar aquí en los problemas de estos puntos de partida, especialmente en lo que respecta al falibilismo ${ }^{6}$ que llevan implícito, pero

\footnotetext{
${ }^{1}$ Sobre esto ya Berkeley, $A$ Treatise Concerning the Principles of human knowledge, I, en 1710 diría "Esse is percipi". Vid. Merleau-Ponty, Phénoménologie de la Perception, Gallimard, Paris 1945; Austin, Sense and Sensibilia (reconstrucción del manuscrito por G. J. Warnock), Oxford University Press, New York, 1964; Anscombe, Aisthesis, Essays in the philosophie of perception, Philosophical Society of Finland, 1976.

${ }^{2}$ Hookway, Truth, Rationality and Pragmatism: Themes from Peirce, Clarendon Press, Oxford 2000, p. 108.

${ }^{3}$ M. H. Fisch et al. (eds.), Writings of Charles S. Peirce: A Chronological Edition, vol. 5, Indiana University Press, Bloomington 1982-2000, p. 258.

4 Respecto de la compatibilidad de esto con las propias afirmaciones de Pierce que pueden encontrarse en Houser et al. (eds.), The Essential Peirce. Selected Philosophical Writings, vol. 2. Indiana University Press, Bloomington 1992-1998; vid. Vargas, Percepto y falibilismo en C. S. Peirce, III Jornadas "Peirce en Argentina", http://www.unav.es/gep/IIIPeirceArgentinaVargas.html.

5 Por eso contra la teoría de las impresiones sensoriales de Pearson, The Grammar of Science, publicada por primera vez en 1892 y con una segunda edición en 1900 publicada por Adam \& Charles Black en Londres, afirma Peirce que son los perceptos los datos iniciales de todo razonamiento y que los objetos físicos son perceptos generalizados. Esto es muy atractivo, pero lo que resulta aún más interesante es dar el paso a otros perceptos generalizados que no son objetos físicos y que a pesar de no tener un "correlato objetivo" adquieren una cierta "existencia" a partir de esa generalización.

6 Pues si bien en las Harvard Lectures de 1903 (Houser et al. (eds.), The Essential Peirce. Selected Philosophical Writings, vol. 2, p. 204) Peirce afirmaba que todo nuestro conocimiento descansaba en
} 
REJ - Revista de Estudios de la Justicia - No 16 - Año 2012

claramente abren la puerta al reconocimiento de que se abren dos flancos a partir de esto. Por una parte, los problemas que presenta nuestra propia percepción y por la otra los problemas de almacenamiento y revisión en nuestra memoria de esos perceptos.

Sin embargo, y a pesar de lo que puede imaginarse luego de esta diatriba, no se trata de retazos arbitrarios de una discusión álgida de carácter filosófico, sino que anticipo que aquí subyace el epicentro de nuestro problema. Partamos por la pregunta más básica que uno se hace frente a estas cuestiones -y que claramente no podremos dilucidar aquí-: dónde está el percepto. Si bien hablamos de lo percibido ello no puede estar fuera, ahí donde está el objeto percibido, porque entonces ese es el objeto de percepción y lo que se percibe no es lo mismo que lo que está afuera. Y no es lo mismo porque está mediado. Está mediado cuando menos por los sentidos y por nuestras capacidades de procesamiento y perspectiva. ${ }^{7}$ Digo cuando menos porque volveremos sobre aquello que se pone en medio del objeto y el sujeto.

Si está mediado por sentidos quiere decir que ya no coincide con lo que está allá afuera porque los sentidos tienen limitaciones y esas limitaciones producirán una diferencia entre el objeto y aquello que está en el sujeto observador. Pero tampoco puede estar completamente adentro. Algunas vertientes constructivistas radicales entienden que ese percepto no es sino una pura producción interna en que el estímulo externo es solo un detonante, una forma de energía que permite que internamente se construya un objeto que en realidad solo existe en la mente del perceptor. ${ }^{8}$

Obviamente no podremos aquí siquiera aproximarnos a una solución respecto de dónde en realidad está el percepto, sino que lo importante es qué hacemos con él. Como hacía presente von Foerster hasta la saciedad en "Observing Systems" (Sistemas que observan) en 1981, es evidente que no hay observación sin observadores y que esos observadores conocen la realidad a partir de sus propias capacidades perceptivas y asociativas.

los juicios perceptuales y que ellos eran veraces e indisputables, en el año anterior (Hartshorne/Weiss /Burks (eds.), Collected Papers of Charles Sanders Peirce, vol. 2, Harvard University Press, Cambridge, MA, 1931-1958, p. 143), señalaba que los hechos perceptuales, en tanto registro intelectual de perceptos, eran falibles. Sobre esto Vargas, ibíd.

${ }^{7}$ Maravillosamente lo describe Merleau-Ponty, Phénoménologie de la Perception, p. 119.

8 Sobre esto Watzlawick, Wie wirklich ist die Wirklichkeit? Wabn, Tauschung, Verstehen, R. Piper \& Co. Verlag. Múnich, 1976 (Hay versión en español, ¿Es real la realidad? Confusión, desinformación, comunicación, Herder, Barcelona, 1979); Von Glassersfeld, Radikaler Konstruktivismus - Ideen, Ergebnisse, Probleme, Suhrkamp, 1996, pp. 96 y ss. Para los alcances jurídicos, vid. Strauch, Wie wirklich sehen wir die Wirklichkeit. Vom Nutzen des Radikalen Konstruktivismus für die juristische Theorie und Praxis, en JZ 21/2000, pp. 1020-1029.

${ }^{9}$ Von Foerster, Observing Systems, Intersystems Publications, Seaside California, 1981. 
Piña - Percepción y decisión. Consideraciones teóricas...

Para usar solo un ejemplo, es como mostrarle un paño azul a una vaca y esperar que luego pueda distinguirlo de otro paño verde. La construcción del mundo de la vaca carece de una distinción perceptiva en este campo porque la vaca ve en blanco y negro. En el percepto el color no existe y por ende no participa en su construcción del mundo. Para ella el mundo carece de color y no es relevante cuánto esfuerzo pongamos en "convencerla" de que los colores existen y sirven para distinguir las cosas.

Pero entonces algún majadero podría preguntar: ¿de verdad existen los colores? Por supuesto que no interesa aquí la discusión acerca de si los colores existen en la realidad o que por no ser más que una propiedad de la luz (su longitud de onda) solo existen como percepto para observadores. ${ }^{10} \mathrm{Da}$ igual. Para los efectos que aquí interesan no parece relevante, porque ese observador [la vaca] no puede tomarlos como un referente para sus decisiones y por ello en su mundo, que es el mismo nuestro - a veces coincidimos con las vacas-, pero que también es distinto - no vivimos en el mundo de las vacas-, los colores no existen.

$\mathrm{Si}$ pensamos en un ejemplo menos pastoril nos podemos aproximar al problema. Imaginemos un caso típico de legítima defensa putativa por ausencia de agresión ilegítima. Un sujeto cree estar siendo agredido en la calle por parte de un tercero que lo quiere abrazar porque lo ha confundido.

Cuando juzgamos las lesiones recibidas por el agresor aparente nuestro sistema opta por eximir de castigo a quien cree que se defiende (salvo que hubiese sido negligente en la decodificación del contexto). La dogmática se escinde tratando de explicar si lo que hay ahí es un error de tipo o un error de prohibición. Para no tomar postura en esta cuestión técnica, digamos que en términos simples el error del sujeto (que lo hizo creer invenciblemente que lo estaban agrediendo) opera como obstáculo a cualquier sanción. Las explicaciones surgidas a partir de estas interrogantes darían pie a acaloradas discusiones entre penalistas, pero eso es lo que no debe perderse de vista: que tendrían lugar "entre penalistas". Esto no quiere decir que sea irrelevante (sabemos que las consecuencias de ello son diversas), pero quiere decir que estamos observando el hecho "cargados conceptualmente" y con un sesgo de perspectiva (la de "los penalistas"). Descarguémoslo de todos esos conceptos y veamos otra manera de ponerlo. En ese hecho se ha producido un desacoplamiento entre el mundo del agresor (que se cree agredido) y el mundo que nosotros creemos que es el real. $\mathrm{Y}$ ese desacoplamiento - cuando no se atribuye al dolo o a la negligencia del imputado-, es un hecho que al Derecho no puede resultar indiferente. Porque ese sujeto actuó como si estuviera en otro mundo. Un mundo en el que lo estaban agrediendo. Dónde estaba ese mundo - afuera o adentro- del sujeto no parece relevante, porque es la misma discusión acerca de dónde está el percepto. Lo importante es que no podemos hacer como si ese mundo particular, propio del sujeto no hubiera

\footnotetext{
${ }^{10}$ Incluso con una potente incidencia cultural según Berlin/Kay, Basic color terms: their universality and evolution, University of California Press, Berkeley/Los Angeles, 1969.
} 
existido porque su toma de decisiones solo se explica por la existencia de ese mundo personal. En otros términos, ese mundo, de algún modo, sí ha existido.

En otros momentos de la historia, como cuando el principio de culpabilidad no se había reconocido como estructura de legitimidad, esta discusión era irrelevante. Cuando el hecho, con independencia de la disposición subjetiva del agente, como decía Achter, producía "una grieta en el edificio del mundo" " no cabía siquiera preguntarse por ese mundo particular, por lo que el sujeto sabía o no sabía, por lo que el sujeto quería o no quería. Pero en nuestro sistema esto ya no es así. Por eso somos capaces de reconocer la existencia de ese mundo y que la decisión que toma ese sujeto tiene pleno sentido en ese mundo (exonerando al responsable) si bien ello carece de todo sentido en este mundo.

Para juzgar el hecho tuvimos que reconocer que había un mundo dentro del mundo y que los que tomaron decisiones conforme a ese otro mundo no son desquiciados, desadaptados, ni nada por el estilo. Simplemente decodificaron y construyeron según sus propias posibilidades y alcances. Ese es el mundo para ellos. ${ }^{12}$

Luego, reconocemos como válida (o cuando menos dotada de un sentido) la decisión adoptada tomando ese mundo como referencia. No es un sinsentido. No se trata de un sujeto que camina y que sin razón alguna golpea a un sujeto con el que interactúa por primera vez y que le está dando la espalda. En ese caso afirmamos que es nuestro mundo el que está vigente y su acción por tanto se estima discordante con el sistema normativo que lo rige, por eso conforme a nuestras reglas actuamos en consecuencia y lo sancionamos. Este caso es claramente diferente.

\section{La mediación colectiva}

Hasta aquí hemos revisado el asunto a nivel individual, es decir, como un error individual de un sujeto contra el mundo. Pero es evidente que colectivamente opera del mismo modo. No solo porque un error colectivo es posible sino muy especialmente porque los colectivos son un lugar muy adecuado para que se creen estos mundos paralelos. En un proceso autorreferente es muy posible que un error compartido termine profundizándose progresivamente hasta producir un

\footnotetext{
11 Achter, Die Geburt der Strafe, V. Klostermann, Frankfurt a.M., 1951, pp. 53 y ss.

${ }^{12}$ En el plano de la teoría de los sistemas y específicamente para explicar la autorreferencia, Ladeur, The theory of autopoiesis as an approach to a better understanding of postmodern law - From the bierarchy of norms to the heterarchy of changing patterns of legal inter-relationships, EUI working papers $n^{\circ} 99 / 3$, ponía el ejemplo del ciego que circula por el mundo ayudado de un bastón. Con él y un par de códigos muy básicos, como firme/endeble o libre/ obstáculo construye su mundo. ¿Está equivocado el ciego en su parcialidad cognitiva? No. Construye sobre lo que puede y vive conforme a ello.
} 
Piña - Percepción y decisión. Consideraciones teóricas...

desacoplamiento completo con la realidad, esto es, un espiral de desacoplamiento. ${ }^{13}$

En ese sistema autónomo de realidad, es decir, en esa realidad propia, se tomarán decisiones conforme al sistema normativo interno. ${ }^{14}$ Esto quiere decir que ellas solo tendrán sentido dentro de ese sistema y para el resto del mundo no lo tendrán.

Con todas estas prevenciones iniciales hago una pregunta particularmente sistémica: ¿las decisiones tomadas en esos marcos (sean individuales o colectivos) tienen o no tienen sentido?

Tres respuestas posibles:

a. Nunca tienen sentido, pues la realidad es la que fija el sentido y ellas no se condicen con la realidad.

b. Siempre tienen sentido autónomamente aunque estén desacopladas con la realidad.

c. Tendrán o no tendrán sentido si existe un marco de referencia que se los ofrezca, sea este la propia realidad o el subsistema en que se encuentra inserto.

Pero a pesar de que no podemos siquiera intentar definir aquí "el sentido" -de hecho, el sentido es indefinible porque para hacerlo se necesita sentido- ${ }^{15}$ sí podemos decir que no es sino una referencia. Algo que dota a la decisión de una consistencia consigo misma o con otro hecho. ${ }^{16}$ Por eso probablemente la posibilidad correcta sea la tercera.

Como ya hemos visto, nuestro sistema de imputación parece reconocer la relevancia de algunos errores que se decanta por esta última posibilidad. Es posible que ellas tengan sentido conforme un sistema autónomo de realidad construida. Si eso es relevante, como cuando declaramos que el error era relevante, no podemos reprochar esa decisión.

\footnotetext{
${ }^{13} \mathrm{E}$ incluso pueden llegar, a partir de iteraciones, a institucionalizarse. Sobre esto Searle, La construcción de la realidad social, Paidós, Barcelona 1997, pp. 93 y ss.

${ }^{14}$ En este punto, desde otra perspectiva, se ha generado una "intencionalidad colectiva". Ibid., pp. 43 y ss. E incluso llegarán a formar parte del mundo "físico". Ibid., pp. 131-132.

${ }^{15}$ Cfr. Luhmann, Soziale Systeme. Grundriss einer allgemeinem Theorie, Suhrkamp, Frankfurt, 1984, p. 93.

${ }_{16}$ Luhmann, Soziologische Aufklärung 1. Aufsätze zur Theorie sozialer Systeme, Westdeutscher Verlag, Opladen 1970, p. 74, lo pone en los siguientes términos: "El mundo es extremadamente complejo. En cambio, las posibilidades de atención de la experiencia y de la acción son muy pequeñas. Esa es la brecha que hay que superar mediante la constitución del sentido". Vid., además, Corsi/Esposito/Baraldi, GLU, Glossar zu Niklas Lubmann Theorie sozialer Systeme, Suhrkamp. Frankfurt a.M. 1997, pp. 170 y ss.; Willke, Systemtheorie I: Grundlagen (6 ${ }^{\mathrm{a}}$ ed.), Lucius \& Lucius, Stuttgart 2000, pp. 40 y ss.
} 
REJ - Revista de Estudios de la Justicia - No 16 - Año 2012

Primera conclusión:

Las decisiones no siempre se toman según lo que pasa (la realidad), sino muchas veces - por nuestros defectos perceptivos- a partir de lo que parece que pasa. No cabe siquiera preguntarse si ello es correcto o no. Tampoco si ello es corregible o no. Para ello se requiere un curso de moral o de antropología social. Para lo que a nosotros nos importa hay que tomarlo como un dato.

\section{Otros problemas}

Luego, todo sujeto con limitaciones cognitivas toma sus decisiones en parte esclavizado por esas limitaciones. Eso nos pasa a los seres humanos.

Esto además enfrenta una nueva complicación -a modo de excurso porque no lo podemos profundizar aquí-, porque nuestros defectos cognitivos no son solo de precepción, es decir, las limitaciones de nuestros sentidos no son la única razón por la que tomamos decisiones desacopladas de la realidad. Además hay otras, como los llamados sesgos cognitivos que han identificado claramente desde la vereda de la economía conductual autores como Kahnemann y Tversky. ${ }^{17}$ Ellos han logrado demostrar, por ejemplo, que muchas veces tomamos decisiones equivocadas no porque tengamos problemas de información (como es el caso de los defectos de percepción), sino porque no somos capaces de decodificarla adecuadamente y cometemos errores sistemáticos. Solo a modo de ejemplo, cuando un sujeto gana la lotería generalmente deja de jugar porque las probabilidades de volver a ganarla son estadísticamente bajas. Esa es una decisión sesgada por el intento de decodificar principios del azar que generalmente se repite en este y otros ámbitos respecto de quienes toman lo que en behavioral economics se denomina decisiones sometidas a riesgo. Sin embargo, basta un minuto de detención para darse cuenta de que las posibilidades de ganar la lotería en el próximo sorteo son idénticas pues ellas dependen del número de participantes, por ejemplo $1 / 5000$ (si hubiera cinco mil participantes). ¿Se reduce esa posibilidad por el resultado del sorteo de la semana pasada? Es evidente que no, pero es común actuar intuitivamente (y erradamente) en ese sentido. Este ya no es un problema de información (la que tenemos es correcta) sino que es un problema de decodificación, de procesamiento de la información. Sin bien no es éste el asunto que hoy nos convoca directamente, para entender el procesamiento de los datos

\footnotetext{
17 Representativo, pero solo a modo de ejemplo, vid. Tversky/Kahnemann, Judgement under uncertainty: heuristics and biases, Science 185, 1974; los mismos, Subjective probability: a judgement of probability, Cognitive Psychology 3, pp. 430-454; los mismos, Prospect Theory. An Analysis of decision under risk, en Econometrica 47, 1979, pp. 263-291; los mismos, The framing of decisions and the psychology of choice, en Science vol. $211 \mathrm{n}^{\circ}$ 4481, pp. 453-458. Además Kahnemann/Knetsch, Anomalies. The endowment effect, loss aversion and statu quo bias, The Journal of economic perspectives 5, $\mathrm{N}^{\circ}$ 1, pp. 193-206.
} 
Piña - Percepción y decisión. Consideraciones teóricas...

que entrega la percepción para la toma de decisiones, también es necesario considerar estos sesgos.

\section{Construcción social de la realidad}

Pero volvamos a nuestra cuestión principal: la toma de decisiones no se hace según lo que pasa en la realidad sino según lo que parece que pasa. La construcción de lo que parece que pasa (o lo que nosotros creemos que pasa) se realiza a través de un conocimiento imperfecto y además procesado imperfectamente. Esto no debe entenderse en sentido de que vivamos en las penumbras dando todo el tiempo palos de ciego. En general, estas condicionantes están acotadas y a pesar de ellas logramos desenvolvernos con bastante naturalidad (si bien lo que entendemos por naturalidad o normalidad está también construido y adolece de los mismos defectos anteriores).

Pero una vez que hemos asumido todo esto llegamos al núcleo de nuestra cuestión: qué incidencia tiene la percepción de algo en la decisión que se toma respecto de ese algo. Una incidencia crucial porque es el primer elemento que se toma en cuenta para la construcción del conocimiento y la generación de conceptos. Por eso Kant decía que los conceptos sin las percepciones están vacíos y que las percepciones sin conceptos son ciegas. ${ }^{18}$ Luego se trata del mismísimo percepto que luego de su procesamiento - con todos sus sesgos- se toma como marco de referencia para la decisión.

Pongamos un ejemplo atingente: una persona decide instalar una alambrada o un cerco eléctrico en su casa para intentar contener la presencia de ladrones o para disuadirlos. Cuántos de los sujetos que toman esa decisión efectivamente han leído alguna estadística georreferenciada de victimización que le permita evaluar adecuadamente la necesidad de hacerlo. Sin duda el porcentaje, si no llega a cero, será muy bajo. El decisor construye la realidad con retazos de información deficientemente recibida (lo que comentan sus vecinos, la experiencia de alguno de ellos, lo que dicen las noticias, etcétera). No es relevante la evaluación de este aspecto, ese no es el punto, lo relevante es que los individuos tomamos la decisión de ese modo construyendo una realidad a base de información deficiente, intuiciones, sistemas propios de creencias, en fin, lo que sea, que es por así decirlo "nuestra realidad", y que puede estar desacoplada a la realidad (ontológica si se quiere). No se trata de sinsentido, todo lo contrario, lo que pasa es que es una decisión que tiene lugar en un contexto diferente, en un mundo dentro del mundo, y en ese sistema de reglas encuentra "su" sentido. Puesto de otra forma, no es un sinsentido, solo que tiene "otro" sentido. La decisión se ha tomado sobre la base de percepción e intuición, construyendo un contexto que le confiere sentido.

\footnotetext{
18 Kant, Crítica de la razón pura, en la Introducción de la Segunda Parte (Idea de una lógica trascendental, I). [N. del A.: Se cita de este modo para evitar los problemas de la multiplicidad de ediciones de una obra de esta envergadura.]
} 
REJ - Revista de Estudios de la Justicia - No 16 - Año 2012

Pero ojalá los problemas terminaran ahí. En realidad recién comienzan. Porque luego a partir de esas decisiones comienzan a tomar decisiones los demás. A partir de la conducta de Ego comienza a tomar decisiones Alter, comienza a esperar cosas (a tener expectativas) y a actuar en consecuencia. Las decisiones se toman entonces conforme a expectativas y recursivamente orientan nuevas decisiones. ${ }^{19}$ No desde las mismas premisas, a esto se referían en gran parte Berger y Luckmann cuando hablaban de "la construcción social de la realidad". ${ }^{20}$

Por eso la percepción de inseguridad, con independencia de su correlato en la realidad, orienta las decisiones de los ciudadanos. ${ }^{21}$

Pero eso no es todo, pues así como esa percepción de inseguridad orienta las decisiones de los que se sienten potenciales víctimas, también orienta la decisión de los potenciales autores de delito. Por eso si se asienta la percepción de que la inseguridad y descontrol es lo que prima en la autodescripción de la sociedad, encontrará menos razones para refrenar su cometido delictivo ${ }^{22}$. Cuando parece que hay inseguridad social reaccionan en consecuencia los que quieren evitarla y también los que quieren aprovecharse de ella.

Precisamente por esto en el diseño de políticas públicas nunca debe menospreciarse esa sensación confrontándola despectivamente con "los números duros". Esto es muy común, decir que las decisiones son equivocadas porque los números demuestran que la realidad es diferente. Ello operaría en una sociedad en que todo ser decisor, efectivamente estudiara las probabilidades estadísticas de su contingencia y no simplemente las intuyera de la información que obtiene del entorno. Volvería a afirmarse, contra los hechos, que los ciudadanos somos el homo oeconomicus perfecto. Sin embargo, en esa sociedad los sujetos pasarían más tiempo juntando antecedentes para tomar la decisión correcta que las horas que tiene el día para tomarlas.

A modo de conclusiones y con el solo objeto de sentar bases para una discusión:

\footnotetext{
${ }^{19}$ Cfr. Luhmann, Sinn als Grundbegriff der Soziologie, en Luhmann/Habermas, Theorie der Gesellschaft oder Sozialtechnologie, Suhrkamp, Frankfurt a.M., 1982, p. 63.

${ }^{20}$ Berger/Luckmann, The social construction of reality, Doubleday \& Co., Nueva York, 1967.

${ }^{21}$ Lo que no justifica que también el Estado opere de ese modo, sea incriminando, sancionando o relajando garantías procesales. El Estado debe considerar estos hechos como un "dato" para la elaboración de políticas públicas, pero no puede responder a ellos sin más sin participar en el afianzamiento de esas percepciones o incluso su espiral de crecimiento. Por esto puede decirse que el diseño de políticas públicas tiene otro estándar (al que no se puede renunciar a pesar de los esfuerzos que continuamente hacen los diseñadores por demostrar que en estas materias sólo se legisla intuitivamente).

22 Vid. Sobre esto y la incidencia del acervo de conocimiento en la vida cotidiana, Schutz/Luckmann, Las estructuras del mundo de la vida, Amorrortu Editores, Buenos Aires 2003, pp. 109 y ss.
} 
Piña - Percepción y decisión. Consideraciones teóricas...

1. El desacoplamiento de la percepción con la realidad no solo es frecuente sino que en gran parte es inevitable.

2. Las decisiones generalmente se toman sobre la base de esas percepciones procesadas según sistemas cognitivos deficientes y sesgados.

3. La construcción social puede propiciar el espiral de desacoplamiento entre percepción de la realidad social y los hechos sociales contrastables.

4. A partir del resultado de ello, se obtiene una realidad que es la realidad en la que cada uno de nosotros vive, a veces acoplado con otros a veces solo. Ello implica que decisiones como protegerse de un delito, cometer un delito, perseguir un delito están fuerte e indisolublemente vinculadas a la percepción social respecto de esos hechos y no a los datos duros que condensan la información acerca de ellos.

5. A partir de esas decisiones se enlazan nuevas decisiones en la vida social y ellas se estructuran y reproducen recíprocamente en un entramado de relaciones que vuelven a generar una realidad propia. $\mathrm{Y}$ ahora, es la realidad social la que orienta las decisiones, y ella incluso formada a partir de una percepción errada (en este caso "de inseguridad) no es menos real que ninguna otra. 
REJ - Revista de Estudios de la Justicia - No 16 - Año 2012

\section{BIBLIOGRAFÍA}

* ACHTER, V.: Die Geburt der Strafe, V. Klostermann, Frankfurt a.M., 1951.

- ANSCOMBE, E.: Aisthesis, Essays in the philosophie of perception, Philosophical Society of Finland, 1976.

* AUSTIN, J.: Sense and Sensibilia (reconstrucción del manuscrito por G. J. Warnock), Oxford University Press, New York, 1964.

* Berger, P./LUCKMANN, T.: The social construction of reality, Doubleday \& Co., Nueva York, 1967.

* BERLIN, B./KAY, P.: Basic color terms: their universality and evolution, University of California Press, Berkeley/Los Angeles, 1969.

* CORSI, G./ESPOSITO, E./BARALDI, C.: GLU, Glossar zu Niklas Lubmann Theorie sozialer Systeme, Suhrkamp. Frankfurt a.M., 1997.

* FISCH, M. H. (ed.): Writings of Charles S. Peirce: A Chronological Edition, vol. 5, Indiana University Press, Bloomington, 1982-2000.

* HOOKWAY, J.: Truth, Rationality and Pragmatism: Themes from Peirce, Clarendon Press, Oxford, 2000

* Houser, N. (ed.): The Essential Peirce. Selected Philosophical Writings, vol. 2. Indiana University Press, Bloomington, 1992-1998.

* KAHNEMANN, D./KNETSCH, J.: "Anomalies. The endowment effect, loss aversion and statu quo bias", The Journal of economic perspectives $5, \mathrm{n}^{\circ} 1$.

* KANT, E.: Crítica de la razón pura, en la Introducción de la Segunda Parte (Idea de una lógica trascendental, I). [N. del A.: Se cita de este modo para evitar los problemas de la multiplicidad de ediciones de una obra de esta envergadura.]

* LADEUR, K.: The theory of autopoiesis as an approach to a better understanding of postmodern lawFrom the hierarchy of norms to the heterarchy of changing patterns of legal inter-relationships, EUI working papers $n^{\circ} 99 / 3$.

* LUHMANN, N.: Soziale Systeme. Grundriss einer allgemeinem Theorie, Suhrkamp, Frankfurt, 1984.

Soziologische Aufklärung 1. Aufsätž zur Theorie sozialer Systeme, Westdeutscher Verlag, Opladen 1970.

"Sinn als Grundbegriff der Soziologie", en Luhmann/Habermas, Theorie der Gesellschaft oder Sożialtechnologie, Suhrkamp, Frankfurt a.M., 1982.

* MerLeau-PONTY, M.: Phénoménologie de la Perception, Gallimard, Paris 1945.

* PEIRCE, Ch.: Collected Papers of Charles Sanders Peirce, Hartshorne/Weiss /Burks (eds.), vol. 2, Harvard University Press, Cambridge, MA, 1931-1958.

* SCHUTZ. A./LUCKMANN, T.: Las estructuras del mundo de la vida, Amorrortu Editores, Buenos Aires 2003.

* SEARLE, J.: La construcción de la realidad social, Paidós, Barcelona 1997.

* TVERSKY, A./KAHNEMANN, D.: "Judgement under uncertainty: heuristics and biases", Science 185, 1974.

"Subjective probability: a judgement of probability", Cognitive Psychology 3.

"Prospect Theory. An Analysis of decision under risk", en Econometrica 47, 1979.

"The framing of decisions and the psychology of choice", en Science vol. 211 $n^{\circ} 4481$.

* VARGAS, E.: "Percepto y falibilismo en C. S. Peirce", III Jornadas "Peirce en Argentina", http://www.unav.es/gep/IIIPeirceArgentinaVargas.html.

* VON fOERSTER, H.: Observing Systems, Intersystems Publications, Seaside California, 1981.

* VON GLASSERSFELD, E.: Radikaler Konstruktivismus - Ideen, Ergebnisse, Probleme, Suhrkamp, 1996. 
Piña - Percepción y decisión. Consideraciones teóricas...

* WATZLAWICK, P.: Wie wirklich ist die Wirklichkeit? Wahn, Tauschung, Verstehen, R. Piper \& Co. Verlag. Múnich, 1976.

¿Es real la realidad? Confusión, desinformación, comunicación, Herder, Barcelona, 1979.

* WILLKE, H.: Systemtheorie I: Grundlagen (6 ${ }^{\mathrm{a}}$ ed.), Lucius \& Lucius, Stuttgart, 2000. 